\title{
Aerococcus viridans infection presenting as cutaneous vasculitis in an immunocompetent patient
}

\author{
Ashaq Hussain Parrey, Fayaz Sofi, Mushtaq Ahmad, Abid Kuchay \\ Shere-i-Kashmir Institute of Medical Sciences, Srinagar, India
}

\begin{abstract}
Background: Aerococcus viridans organisms are Gram-positive cocci that are widely distributed in hospital environments and room air. These bacteria have infrequently been encountered as human pathogens causing bacteremia, endocarditis and urinary tract infections. The significance of these bacteria may be overlooked due to their fastidious growth, and they are often confused with other strains of streptococci or staphylococci.

Case report: We present a case of Aerococcus viridans manifesting as cutaneous vasculitis in an immunocompetent patient. A 30-year-old female patient was admitted to hospital after two weeks history of fever, chills and papular rash over the limbs and trunk. The clinical diagnosis of vasculitis was made. Investigations revealed elevated leucocytosis $\left(21.7 \times 10^{9} / \mathrm{l}\right)$ with $81 \%$ of neutrophils, and an elevated erythrocyte sedimentation rate or $60 \mathrm{~mm} / \mathrm{h}$. Serum anti-neutrophil cytoplasmic antibodies (ANCAs) were not found. Blood culture showed growth of Aerococcus viridans. Histopathological assessment of skin biopsy revealed cutaneous vasculitis.

Conclusions: To date, no clinical case report of this kind has been reported implicating Aerococcus viridans in cutaneous vasculitis. Increased awareness and more studies of this genus should lead to the identification of its potential role in human infections.
\end{abstract}

Key words: Aerococcus viridans, cutaneous vasculitis.

\section{Introduction}

Infectious agents (bacteria and viruses) are considered to be strongly linked to the development of various types of vasculitis, and increased risk of developing vasculitis probably occurs more often in carriers of specific genes and those exposed to the infectious agent. The Gram positive cocci Aerococcus viridans have infrequently been encountered as human pathogens causing bacteremia, endocarditis and urinary tract infections, but it is widely distributed in hospital environments and room air. In this article we present a case report that is an example of development of vasculitis associated with Aerococcus viridans infection.

\section{Case report}

A 30-year-old female patient was referred to the rheumatological department due to $L$ (grade 1) fever, chills and papular rash over the limbs and trunk lasting the previous two weeks. The patient was admitted to the Rheumatology Division of Internal Medicine Sherei-Kashmir Institute of Medical Sciences with clinical suspicion of vasculitis. The patient had a history of hospital admission three weeks ago for hemorrhoidectomy and received ofloxacin ornidazole for five days in the post-operative period in addition to diclofenac for a few days. Investigations revealed a white blood count (WBC) of $21.7 \times 10^{9} /$ with $81 \%$ of neutrophils and $10.8 \%$ of lymphocytes, amount of platelets $339 \times 10^{9} /$, hemoglobin concentration was $11.7 \mathrm{~g} / \mathrm{dl}$ and erythrocyte sedimentation rate (ESR) was $60 \mathrm{~mm} / \mathrm{h}$, serum fasting and postprandial glucose was normal and urine test (overall and the sediment) was normal.

Blood culture showed growth of Aerococcus viridans sensitive to levofloxacin, imipenem, piperacillin/tazo- 
bactam and amoxicillin/clavulanate. Histopathological assessment of skin biopsy revealed thrombi occluding the lumen of small vessels and neutrophilic infiltrates, with scanty nuclear dust.

Additional laboratory tests were performed and the results were as follows: CANCA - negative, pANCA - negative, antinuclear antibodies (ANA) - negative, 24 hour urinary protein - $170 \mathrm{mg} /$ day, kidney function tests and liver function tests were normal. There was not revealed infection of hepatotrophic viruses such as HCV or HBV. Also the HIV test was negative.

Transesophageal echocardiography was normal with no signs of infectious endocarditis. Chest X-ray and ultrasound of the abdomen were normal.

Diagnosis of cutaneous vasculitis associated with infection of Aerococcus viridans was established. The Chapel Hill Consensus Classification from 2012 did not clearly indicate such type of vasculitis; therefore it was considered single organ vasculitis - SOV (cutaneous vasculitis) or vasculitis associated with systemic disease or probable etiology indicating Aerococcus viridans infection as a potential causative agent.

The patient was managed with piperacillin with tazobactam for two weeks, the skin lesions disappeared, and the patient has stopped having a fever (Figs. 1 and 2).

\section{Discussion}

Aerococcus viridans is frequently isolated as a common airborne organism in hospital environments and as a marine organism causing a fatal disease in lobsters [1]. It was first described as a potential human pathogen in 1967 [2]. In humans, aerococci can be found in a very small number as indigenous inhabitants in the upper respiratory tract and on the skin of normal persons [3]. This organism is generally saprophytic and has rarely been encountered as a human pathogen [4-6]. There are case reports of urinary tract infection with bacteremia and endocarditis caused by Aerococcus viridans $[7,8]$. Since Aerococcus viridans is usually recognized as susceptible to penicillin, the treatment protocol for aerococcal infection is generally started with penicillin or its derivates [9].

\section{Summary}

Even though Aerococcus viridans is rarely associated with human infections, it is a potential causative agent of bacteremia, and can cause urinary tract infection and rarely endocarditis. In the described patients the vasculitis was confirmed by skin biopsy and the success of introduced targeted antibiotic therapy confirms such a diagnosis.
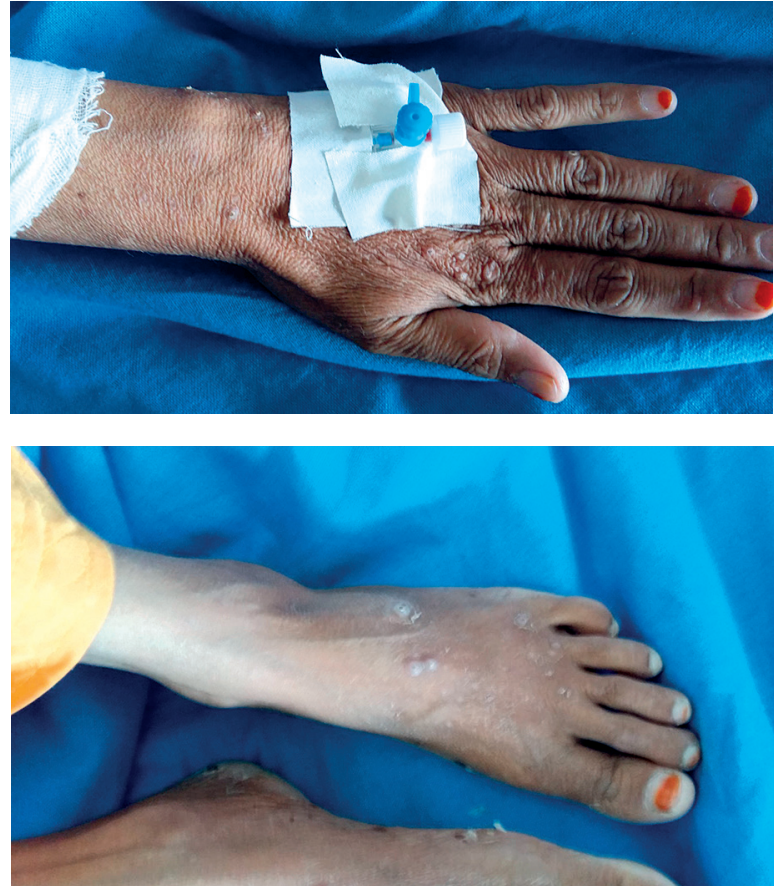

Fig. 1. Patient's hand and feet showing cutaneous papules on fifth day of treatment.

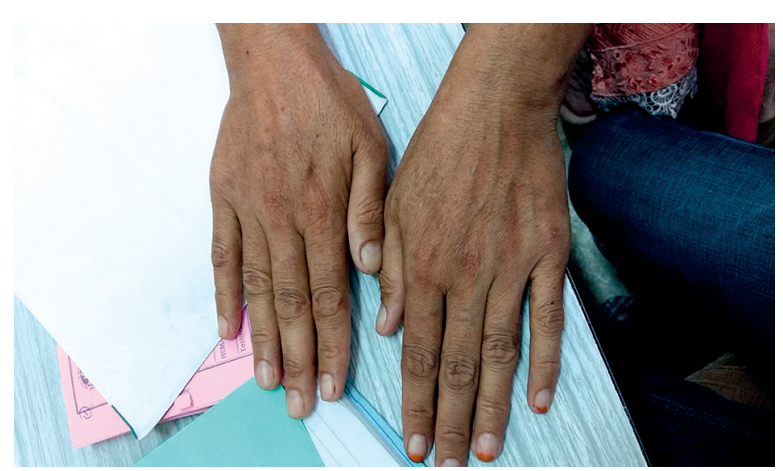

Fig. 2. Patient's hands after two weeks of treatment. Note: The picture baseline was taken on the fifth day of antibiotic treatment when two blood culture reports revealed growth of Aerococcus viridians but not at the initial presentation when cutaneous vasculitis was prominent.

Further investigations are needed to establish the pathogenicity of Aerococcus viridians in immunocompetent people.

The authors declare no conflict of interest.

\section{References}

1. Evans JB. Genus Aerococcus. In: Sneath PH, Mair NS, Sharpe ME, Holt JG (eds.). Bergey's manual of systematic bacteriology. Williams \& Wilkins, Baltimore 1986; 1080. 
2. Colman G. Aerococcus-like organisms isolated from human infections. J Clin Pathol 1967; 20: 294-297.

3. William RE, Hirch A, Cowan ST. Aerococcus, a new bacterial genus. J Gen Microbiol 1953; 8: 475-480.

4. Facklam R, Elliott JA. Identification, classification, and clinical relevance of catalase-negative, gram-positive cocci, excluding the streptococci and enterococci. Clin Microbiol Rev 1995; 8: 479-495.

5. Augustine T, Thirunavukkarasu, Bhat BV, Bhatia BD. Aerococcus viridans endocarditis. Case report. Indian Pediatr 1994; 31: 599-601.

6. Swanson H, Cutts E, Lepow M. Penicillin-resistant Aerococcus viridans bacteremia in a child receiving prophylaxis for sickle-cell disease. Clin Infect Dis 1996; 22: 387-388.

7. Gopalachar A, Akins RL, Davis WR, Siddiqui AA. Urinary tract infection caused by Aerococcus viridans, a case report. Med Sci Monit 2004; 10: CS73-75.

8. Chen LY, Yu WC, Huang SH, et al. Successful treatment of Aerococcus viridans endocarditis in a patient allergic to penicillin. J Microbiol Immunol Infect 2012; 45: 158-160.

9. Pien FD, Wilson WR, Kunz K, Washington JA 2nd. Aerococcus viridans endocarditis. Mayo Clin Proc 1984; 59: 47-48. 